\title{
Long non-coding RNA OTUD6B-AS1 overexpression inhibits the proliferation, invasion and migration of colorectal cancer cells via downregulation of microRNA-3171
}

\author{
WEI WANG $^{1 *}, \mathrm{XIA} \mathrm{CHENG}^{2,3^{*}}$ and JIANHUA ZHU ${ }^{1}$ \\ ${ }^{1}$ Department of Emergency Traumatic Surgery, Shanghai Pudong New District Zhoupu Hospital \\ (Shanghai University of Medicine and Health Sciences Affiliated Zhoupu Hospital), Shanghai 201318; \\ ${ }^{2}$ Graduate School, Dalian Medical University; ${ }^{3}$ Department of Oncology, \\ Affiliated Zhongshan Hospital of Dalian University, Dalian, Liaoning 116000, P.R. China
}

Received June 24, 2020; Accepted October 13, 2020

DOI: $10.3892 / \mathrm{ol} .2021 .12454$

\begin{abstract}
Colorectal cancer (CRC) is a common digestive system malignancy and a major cause of cancer-associated mortality worldwide. Aberrant expression of long non-coding RNAs has been reported in several types of cancer. The aim of the present study was to investigate the role of ovarian tumor domain containing 6B antisense RNA1 (OTUD6B-AS1) in CRC and its underlying mechanisms. OTUD6B-AS1 expression in CRC cell lines was examined using reverse transcription-quantitative PCR. Furthermore, The Cancer Genome Atlas database was utilized to examine the expression levels of OTUD6B-AS1 in CRC tissues. Following OTUD6B-AS1 overexpression, Cell Counting Kit-8 and colony formation assays were used to detect the proliferation ability of HCT116 cells. The expression levels of proliferation-related protein Ki67 were determined using immunofluorescence staining. Subsequently, Transwell and wound healing assays were used to evaluate the invasion and migration of HCT116 cells, respectively. The expression levels of migration-related proteins (MMP2 and MMP9) were measured using western blotting. Additionally, a luciferase reporter assay was used to verify the potential interaction between OTUD6B-AS1 and microRNA-3171 (miR-3171). Subsequently, rescue assays were performed to clarify the regulatory effects of OTUD6B-AS1 and miR-3171 on CRC development. The results demon-
\end{abstract}

Correspondence to: Dr Jianhua Zhu, Department of Emergency Traumatic Surgery, Shanghai Pudong New District Zhoupu Hospital, (Shanghai University of Medicine and Health Sciences Affiliated Zhoupu Hospital), 1500 Zhouyuan Road, Zhoupu, Pudong New Area, Shanghai 201318, P.R. China

E-mail: zhujhua33@126.com

\section{${ }^{*}$ Contributed equally}

Key words: colorectal cancer, ovarian tumor domain containing 6B antisense RNA1, proliferation, invasion, migration strated that OTUD6B-AS1 expression was low in CRC cells and tissues. Overexpression of OTUD6B-AS1 inhibited the proliferation, invasion and migration of HCT116 cells. Furthermore, miR-3171 was demonstrated to be a direct target of OTUD6B-AS1 using a luciferase reporter assay. The rescue assays revealed that miR-3171 mimics markedly reversed the inhibitory effects of OTUD6B-AS1 overexpression on proliferation, invasion and migration of CRC cells. Overall, these findings demonstrated that OTUD6B-AS1 overexpression inhibited the proliferation, invasion and migration of HCT116 cells via downregulation of miR-3171, suggesting that OTUD6B-AS1 may serve as a novel biomarker for CRC treatment.

\section{Introduction}

Colorectal cancer (CRC) was the third most common cause of cancer-associated mortality worldwide, and caused approximately 900,000 deaths in 2013 (1). Due to technological advances in colonoscopy and other screening measures, the health condition of patients with $\mathrm{CRC}$ has improved in recent years. However, it has been estimated that the overall incidence of CRC worldwide will increase by $60 \%$ to $>2.2$ million cases and 1.1 million deaths by 2030 (2). Notably, CRC incidence and mortality rates are increasing rapidly in developing countries (3). Furthermore, the incidence of CRC in the young generation is rising, as epidemiological studies have noted the rising numbers of adolescents and adults $<50$ years old who are diagnosed with this disease $(4,5)$. Genetic and lifestyle factors are the major contributors to the disease; however, the exact pathogenesis of CRC is unknown. Therefore, it is important to identify a novel target for the diagnosis and clinical treatment of CRC.

Long non-coding RNAs (lncRNAs) represent a class of transcripts with a length $>200$ nucleotides, and do not exhibit any capacity to encode proteins (6). IncRNAs are indispensable regulators in the process of gene expression, and emerging evidence has revealed the involvement of lncRNAs in cancer development and progression, since several studies have identified that the aberrant expression of lncRNAs is closely 
associated with biological behaviors of malignant carcinoma cells, such as proliferation, invasion and metastasis $(7,8)$. lncRNA ovarian tumor domain containing 6B antisense RNA1 (OTUD6B-AS1) is oriented in an antisense direction relative to the protein-coding gene OTUD6B on the opposite DNA strand (9). OTUD6BAS1 is located on chromosome 8q21.3 and has 2,179 bp (NR_110439, ENST00000524003.1) (10). A previous study has demonstrated that OTUD6B-AS1 expression is downregulated in clear cell renal cell carcinoma tissue samples, while overexpression of OTUD6B-AS1 inhibits cell proliferation, migration and invasion of clear cell renal cell carcinoma (11). However, to the best of our knowledge, the role of OTUD6B-AS1 in CRC has not yet been determined.

MicroRNAs (miRNAs/miRs) are highly conserved endogenous non-coding RNAs that are 22 nucleotides long. They have been demonstrated to serve important roles in a variety of biological processes, including development, differentiation and signaling (12-15). Dysregulated miRNAs may function as either tumor suppressors or oncogenes in carcinoma by targeting each one of these features (16). Using Starbase, it was predicted that OTUD6B-AS1 can bind to miR-3171. Studies have demonstrated that miR-3171 expression is abnormally increased in bladder cancer and hepatocellular carcinoma tissues $(17,18)$. Therefore, it was hypothesized that OTUD6B-AS1 could exert certain effects on the proliferation, invasion and migration of CRC cells by regulating miR-3171.

To the best of our knowledge, the present study was the first to investigate the role of OTUD6B-AS1 in CRC, and to examine whether OTUD6B-AS1 could affect the proliferation, invasion and migration of CRC cells by regulating miR-3171.

\section{Materials and methods}

The Cancer Genome Atlas (TCGA) database analysis. Human RNA-sequencing data from colorectal cancer projects, which included 482 patients with CRC and 155 normal tissues (project no. TCGA-COAD) were obtained by TCGA (https://portal.gdc. cancer.gov/) analysis in the UALCAN database (ualcan.path. uab.edu/) (19). A Mann-Whitney test was used to determine the statistical significance of the difference in OTUD6B-AS1 expression between normal and tumor samples.

Cell culture. CRC cell lines (Caco2, HCT116, LoVo, SW480 and SNU-C1) and the normal intestinal epithelial cell line (HIEC) were purchased from American Type Culture Collection. All cell lines were routinely maintained in RPMI-1640 medium (HyClone; Cytiva) supplemented with 10\% FBS (Gibco; Thermo Fisher Scientific, Inc.), $100 \mathrm{U} / \mathrm{ml}$ penicillin and $100 \mu \mathrm{g} / \mathrm{ml}$ streptomycin (Invitrogen; Thermo Fisher Scientific, Inc.). Cells were cultured in a humidified incubator at $37^{\circ} \mathrm{C}$ with $5 \% \mathrm{CO}_{2}$. The culture medium was replaced every 3 days. Cells were passaged when $80 \%$ confluence was reached.

Cell transfection. The OTUD6B-AS1 overexpression plasmid (Oe-OTUD6B-AS1; $1 \mu \mathrm{g}$ ) and empty vector (Oe-NC; $1 \mu \mathrm{g}$ ) were obtained from Shanghai GenePharma Co., Ltd. miR-3171 mimics (40 nM; cat. no. miR10015046-1-5) and corresponding scrambled mimic negative control (mimic-NC; $40 \mathrm{nM}$; cat. no. miR1N0000001-1-5) were purchased from Guangzhou RiboBio Co., Ltd. Cells $\left(1 \times 10^{6}\right.$ cells/well) were transfected with the aforementioned oligonucleotides using Lipofectamine ${ }^{\circledR} 3000$ (Invitrogen; Thermo Fisher Scientific, Inc.) according to the manufacturer's protocols at $37^{\circ} \mathrm{C}$ for $48 \mathrm{~h}$. At $48 \mathrm{~h}$ after transfection, HCT116 cells were harvested for further experiments, and successful transfection was verified using reverse transcription-quantitative PCR (RT-qPCR).

$R T-q P C R$. Total RNA was extracted from HCT116 cells using TRIzol ${ }^{\circledR}$ reagent (Invitrogen; Thermo Fisher Scientific, Inc.). Total RNA was then reverse transcribed into cDNA at $42^{\circ} \mathrm{C}$ for 30 min using a reverse transcription kit (PrimeScript ${ }^{\mathrm{TM}} \mathrm{RT}$ Reagent Kit; Takara Bio, Inc.). qPCR was performed using iTaq $^{\mathrm{TM}}$ Universal SYBR ${ }^{\circledR}-$ Green Supermix (Bio-Rad Laboratories, Inc.) on an ABI 7500 instrument (Applied Biosystems; Thermo Fisher Scientific, Inc.). The following thermocycling conditions were used: Pre-denaturation at $95^{\circ} \mathrm{C}$ for $10 \mathrm{~min}$, denaturation at $95^{\circ} \mathrm{C}$ for $15 \mathrm{sec}$ and annealing at $60^{\circ} \mathrm{C}$ for $1 \mathrm{~min}$ (40 cycles). The sequences of the gene-specific primers used in the present study were as follows: IncRNA OTUD6B-AS1 forward, 5'-AGCACACCCAGTCAGAAACCAG-3' and reverse, 5'-TCTACAAACGGGAATGTCG-3'; miR-3171 forward, 5'-AGATGTATGGAATCTGTATATA-3' and reverse, 5'-GAA CATGTCTGCGTATCTC-3'; GAPDH forward, 5'-TGTGGG CATCAATGGATTTGG-3' and reverse, 5'-ACACCATGTATT CCGGGTCAAT-3'; and U6 forward, 5'-TCTGCTCCTATCC CAATTACCTG-3' and reverse, 5'-ACTCCCGGATCTCTTCT AAGTTG-3'. GAPDH and U6 were used as internal controls for OTUD6B-AS1 and miR-3171, respectively, and relative expression was calculated based on the $2^{-\Delta \Delta \mathrm{Cq}}$ method (20).

Cell Counting Kit-8 (CCK-8) assay. HCT116 cells were seeded into a 96-well plate at a density of $2 \times 10^{4}$ cells/well and cultured at $37^{\circ} \mathrm{C}$ with $5 \% \mathrm{CO}_{2}$. Following transfection for 24,48 and $72 \mathrm{~h}, 10 \mu \mathrm{l}$ CCK-8 solution [OBiO Technology (Shanghai) Corp., Ltd.] was added to each well. Following incubation at $37^{\circ} \mathrm{C}$ for $4 \mathrm{~h}$, the absorbance at $450 \mathrm{~nm}$ was detected using a spectrophotometer (Thermo Fisher Scientific, Inc.).

Colony formation assay. The HCT116 cells $\left(0.5 \times 10^{3}\right.$ cells/well $)$ were seeded in a six-well plate and cultured for 10 days after treatment. Subsequently, colonies were fixed with $10 \%$ formaldehyde for $10 \mathrm{~min}$ at room temperature and stained with $0.5 \%$ crystal violet for $5 \mathrm{~min}$ at room temperature. The number of colonies was counted using ImageJ software (version 1.52r; National Institutes of Health) and images were captured under a fluorescence inversion microscope (Olympus Corporation).

Immunofluorescence staining. Treated HCT116 cells were fixed with $4 \%$ paraformaldehyde for $30 \mathrm{~min}$ at $37^{\circ} \mathrm{C}$, and then $0.5 \%$ Triton $\mathrm{X}-100$ was used to permeabilize the cells at room temperature for 20 min. After blocking with 5\% BSA (Beyotime Institute of Biotechnology) for $1 \mathrm{~h}$ at room temperature, slides were incubated overnight at $4^{\circ} \mathrm{C}$ with a primary antibody against Ki67 (cat. no. ab15580; dilution, 1:1,000; Abcam). Subsequently, the slides were incubated with a fluorescent secondary antibody (cat. no. BA1105; dilution, 1:10,000; Boster Biological Technology) for $1 \mathrm{~h}$ in a wet box at room temperature in the dark. Finally, DAPI was used to counterstain the nuclei at room temperature for $15 \mathrm{~min}$. Images were captured under a fluorescence inversion Olympus microscope (Olympus Corporation). 
Luciferase reporter assay. Potential target genes of lncRNA OTUD6B-AS1 were predicted using an online bioinformatics software Starbase 2.0 (http://starbase.sysu.edu.cn/) (21). HCT116 cells were reseeded into 24-well plates and cultured for $24 \mathrm{~h}$. The fragments of OTUD6B-AS1 containing predicted wild-type (WT) and mutant (MUT) miR-3171 binding sequences were amplified by Shanghai GenePharma Co., Ltd., and inserted into the luciferase reporter gene of the pmirGLO vector (Promega Corporation) to produce the reporter plasmids OTUD6B-AS1-WT and OTUD6B-AS1-MUT, respectively. Subsequently, the cells $\left(1 \times 10^{4}\right.$ cells/well $)$ were co-transfected with plasmids and miR-3171 mimic $(40 \mathrm{nM}$; cat. no. miR10015046-1-5; Guangzhou RiboBio Co., Ltd.) or mimic-NC vector (40 nM; cat. no. miR1N0000001-1-5; Guangzhou RiboBio Co., Ltd.) using Lipofectamine ${ }^{\circledR} 2000$ (Invitrogen; Thermo Fisher Scientific, Inc.) for $24 \mathrm{~h}$. At $48 \mathrm{~h}$ after transfection, the culture medium was removed and the cells were rinsed twice with PBS. The HCT116 cells were lysed to obtain cell lysates, which were swirled for $10 \mathrm{~min}$ and centrifuged at $12,000 \mathrm{xg}$ for $10 \mathrm{~min}$ at $4^{\circ} \mathrm{C}$, and the supernatant was transferred to a new Eppendorf tube. According to the instructions of the dual-luciferase reporter assay kit (Beijing Solarbio Science \& Technology Co., Ltd.), the fluorescence value was used as an internal reference, and the fluorescence value was detected using an enzyme marker. The results were normalized to Renilla luciferase activity.

Transwell assay. The invasion ability of HCT116 cells was evaluated using Transwell assay (pore size, $8.0 \mu \mathrm{m}$; Corning Inc.) coated with Matrigel (BD Biosciences) overnight at $37^{\circ} \mathrm{C}$. A total of $2 \times 10^{4}$ HCT116 cells in serum-free medium were seeded into each upper chamber. DMEM containing 10\% FBS was added to the lower chamber as a chemoattractant. After 48 h of incubation at $37^{\circ} \mathrm{C}, 4 \%$ paraformaldehyde was used to fix invasive cells for $30 \mathrm{~min}$ at room temperature and $0.1 \%$ crystal violet was subsequently applied to stain cells for $30 \mathrm{~min}$ at room temperature. Images were obtained under an inverted light microscope and the numbers of invasive HCT116 cells were counted using ImageJ software (version 1.52r; National Institutes of Health).

Wound healing assay. HCT116 cells from each group were collected and seeded into 6-well plates at a density of $1 \times 10^{6}$ cells $/ \mathrm{ml}$. When the cells completely covered the bottom of the well, a vertical line was drawn across the well with a $10-\mu 1$ pipette tip. HCT116 cells were washed with PBS three times to remove cell debris, and subsequently visualized under an inverted microscope. The images were labeled as $0 \mathrm{~h}$. Subsequently, the medium was replaced with serum-free medium and cells were continuously cultured for $24 \mathrm{~h}$. Images were captured under an inverted light microscope and labeled as $24 \mathrm{~h}$. The $0 \mathrm{~h}$ images were used as a reference. The relative cell migration was analyzed using ImageJ software (version 1.52r; National Institutes of Health).

Western blot analysis. The samples were collected from HCT116 cells using RIPA buffer (Beyotime Institute of Biotechnology). Afterwards, the concentration of proteins was measured using the BCA method (Beyotime Institute of Biotechnology). Subsequently, the proteins (40 $\mu \mathrm{g} / \mathrm{lane})$

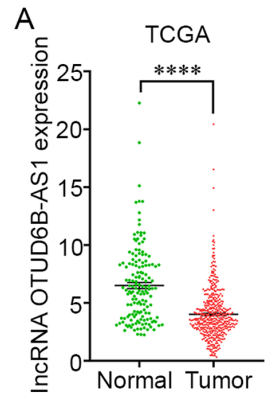

B

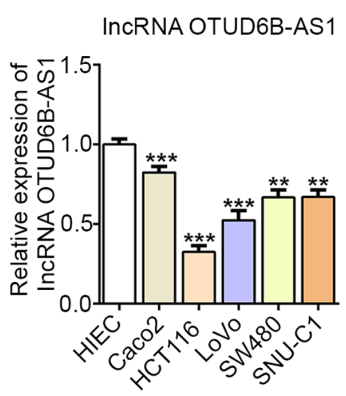

Figure 1. OTUD6B-AS1 is expressed at low levels in CRC tissues and several CRC cell lines (Caco2, HCT116, LoVo, SW480 and SNU-C1). (A) TCGA was used to determine the mRNA expression levels of OTUD6B-AS1 in CRC tumor tissues and adjacent non-tumor tissues. ${ }^{* * * * *} \mathrm{P}<0.0001$ vs. normal. (B) Expression levels of OTUD6B-AS1 in CRC cell lines were measured by reverse transcription-quantitative PCR. ${ }^{* *} \mathrm{P}<0.01$ and ${ }^{* * *} \mathrm{P}<0.001$ vs. HIEC. OTUD6B-AS1, ovarian tumor domain containing $6 \mathrm{~B}$ antisense RNA1; CRC, colorectal cancer; lncRNA, long non-coding RNA; TCGA, The Cancer Genome Atlas.

were separated on a 10\% SDS-PAGE gel (Beyotime Institute of Biotechnology). The proteins were transferred to PVDF membranes (EMD Millipore). The PVDF membranes were blocked with $5 \%$ non-fat milk powder for $1.5 \mathrm{~h}$ at room temperature. Then, the membranes were incubated with primary antibodies at $4^{\circ} \mathrm{C}$ overnight. Primary antibodies, including anti-MMP2 (cat. no. 40994S; dilution, 1:1,000), anti-MMP9 (cat. no. 13667T; dilution, 1:1,000) and the loading control anti-GAPDH (cat. no. 5174T; dilution, 1:1,000), were purchased from Cell Signaling Technology, Inc. On the next day, the membranes were washed with PBS with $0.2 \%$ Tween-20 (PBST) three times and probed with horseradish peroxidase-conjugated secondary antibodies (cat. no. sc-2004; dilution, 1:3,000; Santa Cruz Biotechnology, Inc.) for $1.5 \mathrm{~h}$ at room temperature. Afterwards, the immunoreactive bands were washed with PBST again. Finally, the signal was monitored and visualized using an enhanced chemiluminescence assay (EMD Millipore) and the Odyssey Infrared Imaging system (LI-COR Biosciences). GAPDH was used as an internal control. The intensity of the bands was semi-quantified using ImageJ software (version 1.52r; National Institutes of Health).

Statistical analysis. All experiments were repeated three times independently. Data are presented as the mean \pm standard deviation and were analyzed using GraphPad Prism software (version 6.0; GraphPad Software, Inc.). Comparisons between groups were performed using an unpaired t-test, and multiple comparisons were performed using one-way ANOVA followed by Tukey's post hoc test. $\mathrm{P}<0.05$ was considered to indicate a statistically significant difference.

\section{Results}

OTUD6B-AS1 expression is downregulated in HCT116 cells and CRC tissues. As predicted by TCGA, OTUD6B-AS1 expression was markedly downregulated in CRC tissues compared with in normal tissues (Fig. 1A). Furthermore, the expression levels of OTUD6B-AS1 were assessed in different CRC cell lines and in normal HIEC cells. Compared 
A

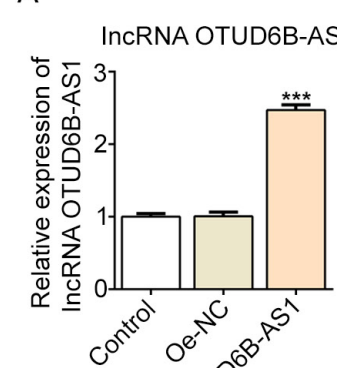

$B$

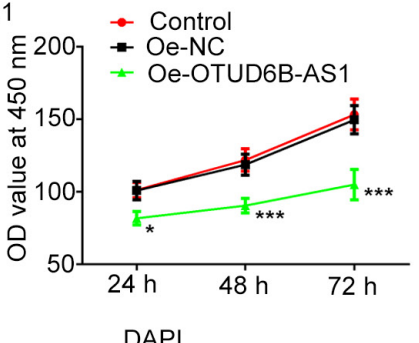

C
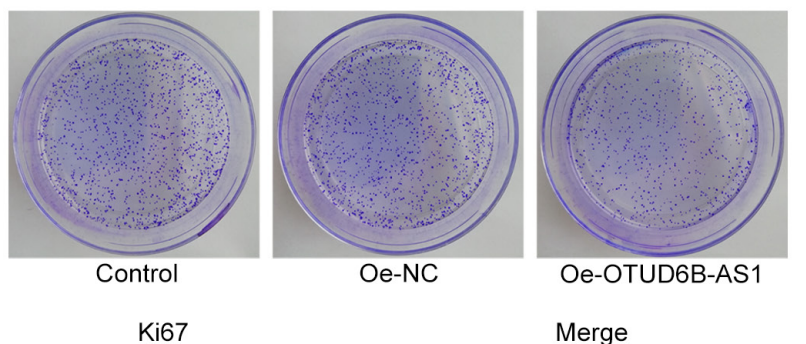

D
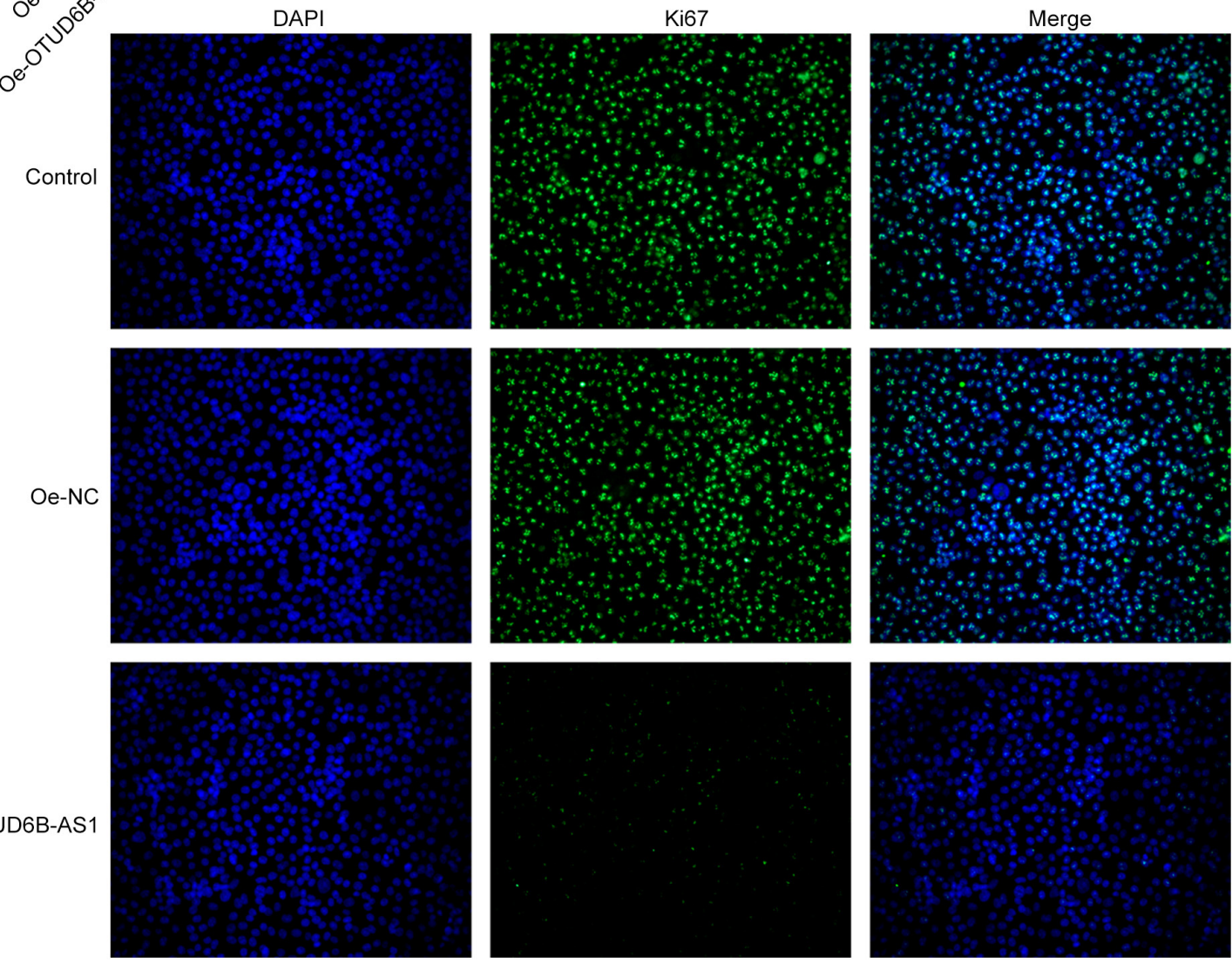

Figure 2. Overexpression of OTUD6B-AS1 inhibits the proliferation of HCT116 cells. (A) mRNA expression levels of OTUD6B-AS1 were determined using reverse transcription-quantitative PCR following transfection with OTUD6B-AS overexpression plasmid. The proliferation of HCT116 cells was measured using (B) Cell Counting Kit-8 and (C) colony formation assays. (D) Expression levels of proliferation-related protein Ki67 were detected by immunofluorescence staining. Magnification, $\mathrm{x} 200$. $^{*} \mathrm{P}<0.05$ and ${ }^{* * *} \mathrm{P}<0.001$ vs. Oe-NC. OTUD6B-AS1, ovarian tumor domain containing $6 \mathrm{~B}$ antisense RNA1; NC, negative control; Oe, overexpression; OD, optical density; lncRNA, long non-coding RNA.

with those in normal HIEC cells, the expression levels of OTUD6B-AS1 in CRC cell lines (Caco2, HCT116, LoVo, SW480 and SNU-C1) were markedly downregulated, particularly in HCT116 cells (Fig. 1B). Therefore, HCT116 cells were selected for subsequent experiments.

Overexpression of OTUD6B-AS1 inhibits the proliferation, invasion and migration of HCT116 cells. After constructing the overexpression plasmid of OTUD6B-AS1, transfection efficiency was verified by RT-qPCR, and it was demonstrated that OTUD6B-AS1 expression was significantly upregulated in the Oe-OTUD6B-AS1 group compared with the Oe-NC group (Fig. 2A). The results of CCK-8 and colony formation assays revealed that the proliferation and colony formation abilities of cells in the Oe-OTUD6B-AS1 group were inhibited compared with those in the Oe-NC group (Fig. 2B and C). Additionally, an immunofluores- cence assay revealed a decrease in the expression levels of proliferation-related protein Ki67 following OTUD6B-AS1 overexpression (Fig. 2D). Furthermore, as shown in Fig. 3A-D, OTUD6B-AS1 overexpression notably suppressed the invasion and migration of HCT116 cells compared with those of cells in the empty vector (Oe-NC) group. Additionally, the expression levels of migration-associated proteins, including MMP2 and MMP9, were decreased in the overexpression group (Fig. 3E). Therefore, it could be concluded that the overexpression of OTUD6B-AS1 suppressed the proliferation, invasion and migration of CRC cells.

miR-3171 is a direct target of OTUD6B-AS1. The binding site between OTUD6B-AS1 and miR-3171 was predicted using Starbase 2.0 (Fig. 4A). Subsequently, RT-qPCR was utilized to detect the expression levels of miR-3171 in CRC cell lines and HIEC cells. It was identified that miR-3171 expression was 

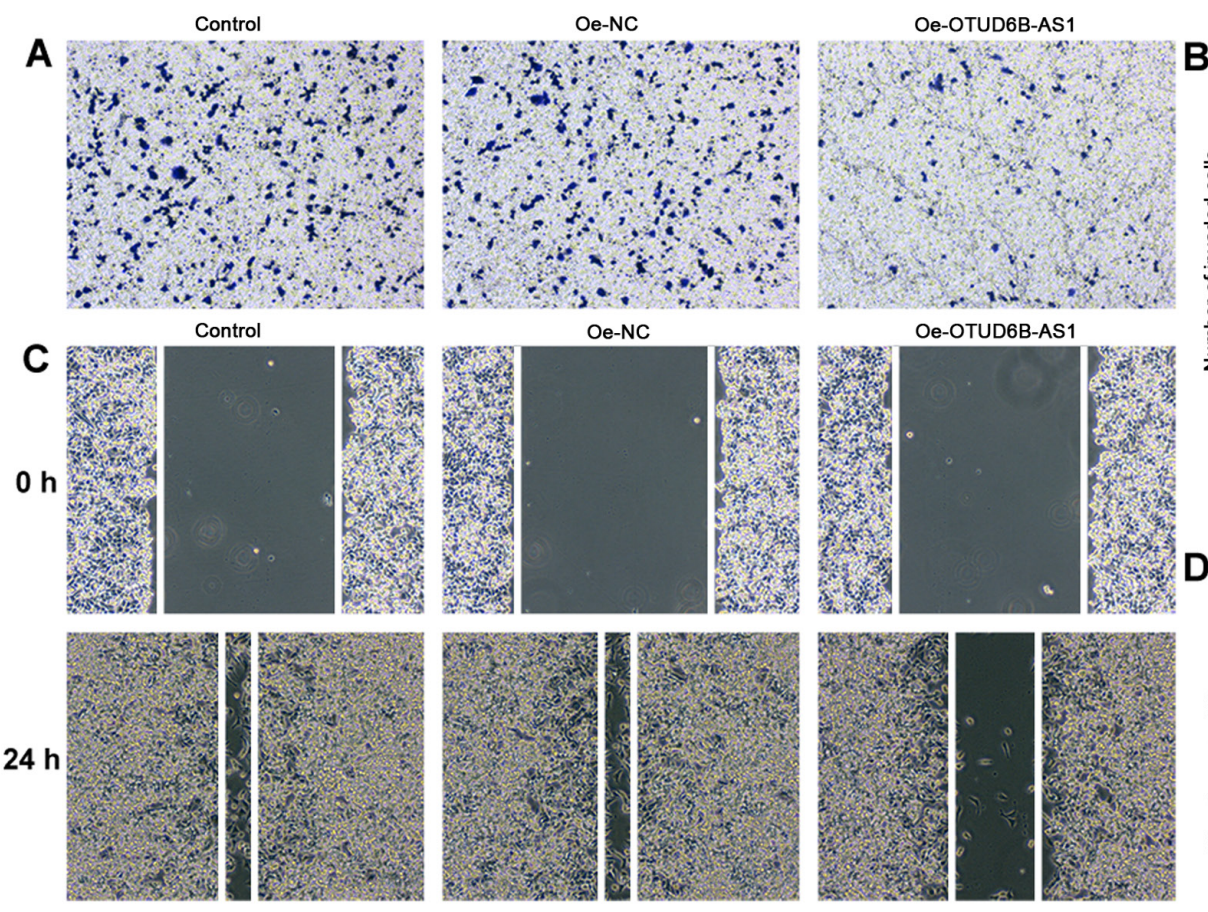

B
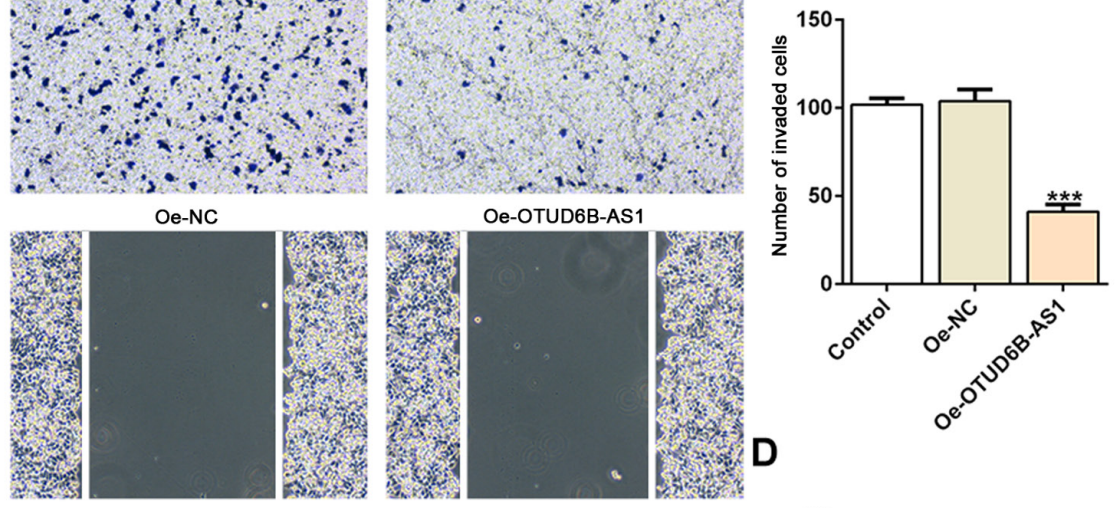

E
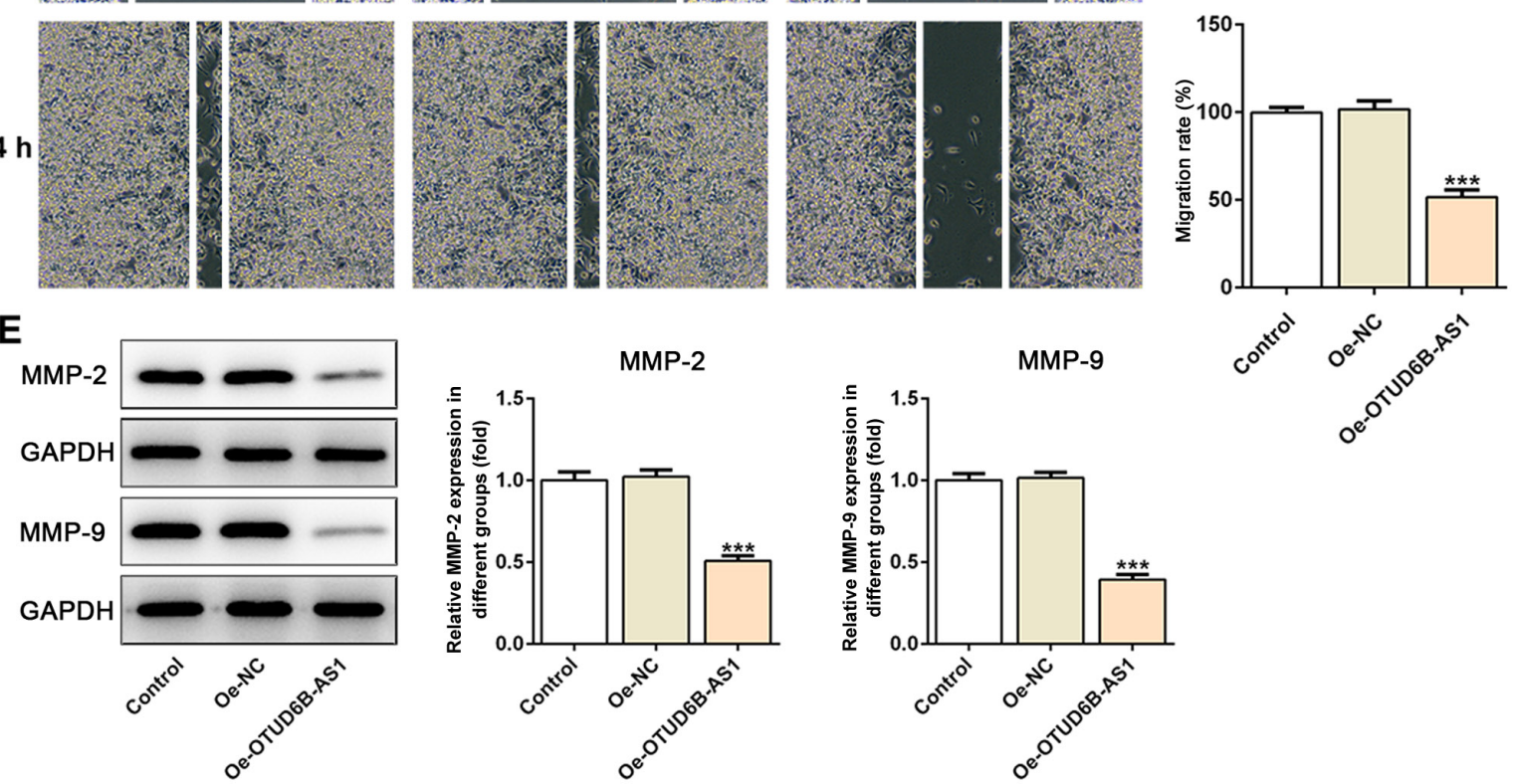

Figure 3. Overexpression of OTUD6B-AS1 suppresses the invasion and migration of HCT116 cells. (A) The invasive ability of HCT116 cells was detected using a Transwell assay. Magnification, x100. (B) Relative number of invaded cells. (C) The migratory activity of cells was evaluated using a scratch wound healing assay. Magnification, x200. (D) Relative migration rate. (E) Expression levels of migration-related proteins were measured by western blot analysis.

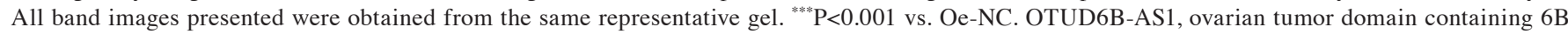
antisense RNA1; NC, negative control; Oe, overexpression.

A

OTUD6B-AS1 : 5' guungggaggGgGUGgAUACAUCc 3'

miR-3171 : 3 ' cuauauaUGUCUAAGGUAUGUAGa 5 ,
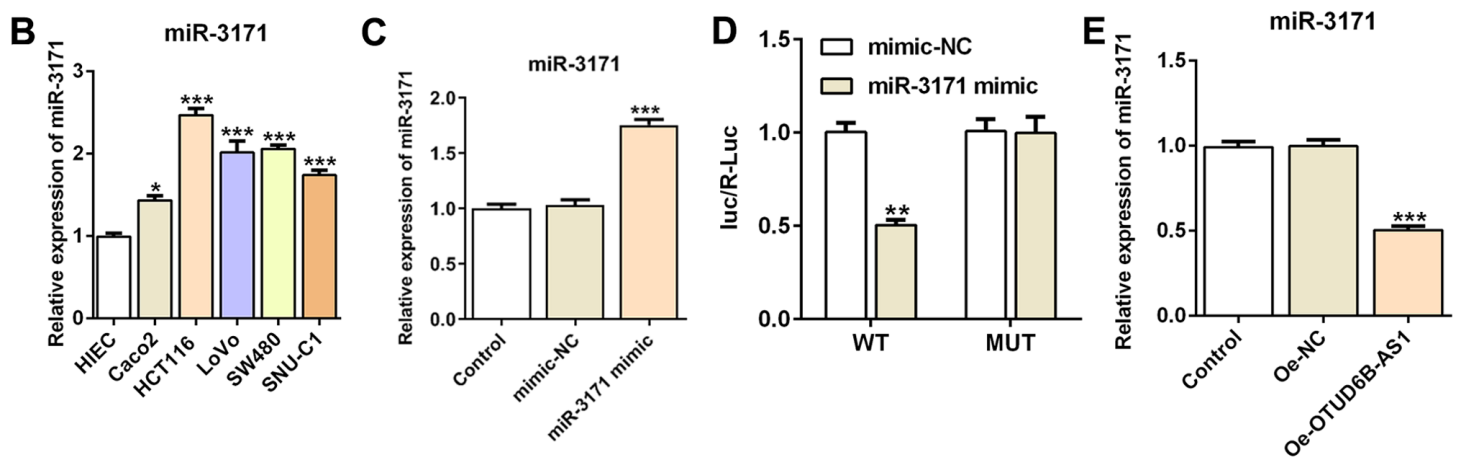

Figure 4. miR-3171 is a direct target of OTUD6B-AS1. (A) Binding region between OTUD6B-AS1 and miR-3171. (B) Expression levels of miR-3171 in colorectal cancer cell lines (Caco2, HCT116, LoVo, SW480 and SNU-C1) were detected by RT-qPCR. "P<0.05, ${ }^{* * *}$ P $<0.001$ vs. HIEC. (C) RT-qPCR was used to evaluate the expression levels of miR-3171 after transfection. ${ }^{* * *} \mathrm{P}<0.001$ vs. mimic-NC. (D) A luciferase reporter assay was performed to detect the relative luciferase activity. ${ }^{* *} \mathrm{P}<0.01$ vs. mimic-NC. (E) RT-qPCR was used to assess the expression levels of miR-3171 following OTUD6B overexpression. ${ }^{* * * *} \mathrm{P}<0.001$ vs. Oe-NC. OTUD6B-AS1, ovarian tumor domain containing 6B antisense RNA1; NC, negative control; Oe, overexpression; WT, wild-type; MUT, mutant; miR-3171, microRNA-3171; RT-qPCR, reverse transcription-quantitative PCR; luc/R-Luc, luciferase activity/Renilla luciferase activity. 
A

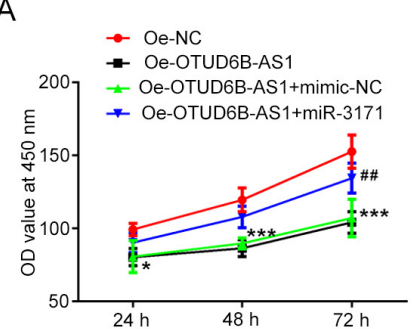

C
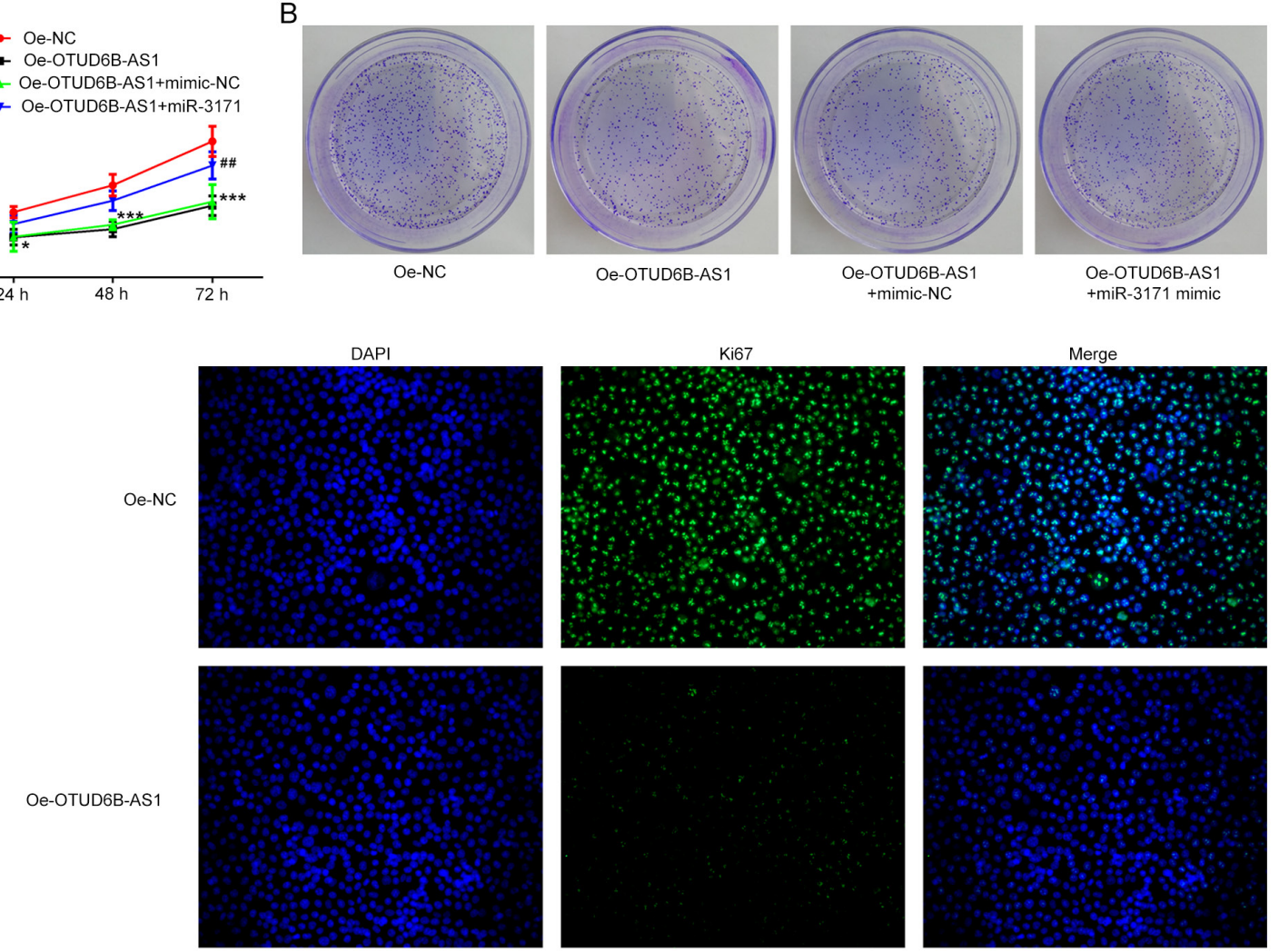

Oe-OTUD6B-AS1
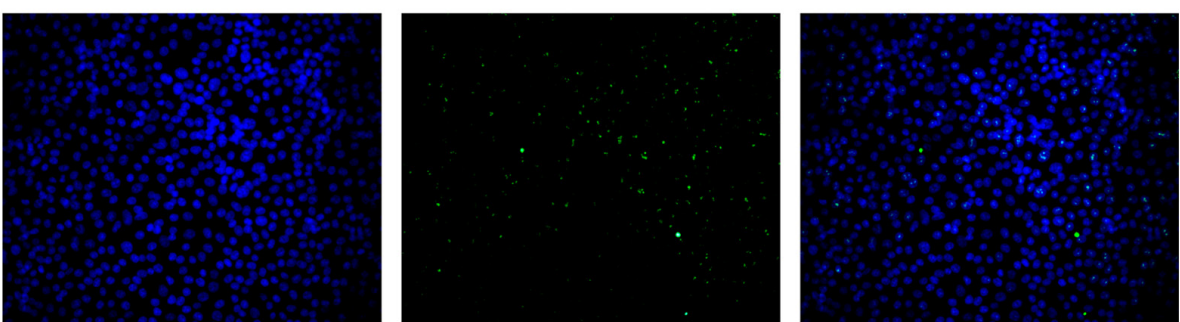

Oe-OTUD6B-AS1 + mimic-NC
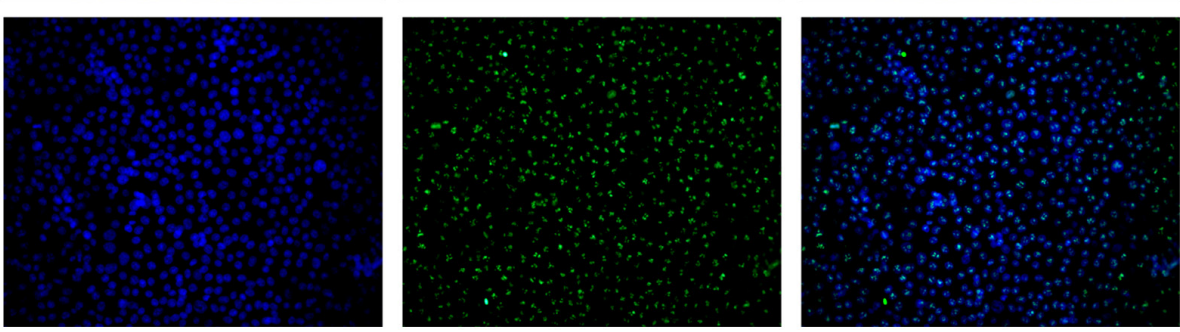

Figure 5. miR-3171 mimic reverses the inhibitory effect of OTUD6B-AS1 overexpression on the proliferation of HCT116 cells. The proliferation of HCT116 cells was examined using (A) Cell Counting Kit-8 and (B) colony formation assays following co-transfection of OTUD6B-AS1 overexpression plasmid and miR-3171 mimic. (C) Expression of proliferation-related protein Ki67 was detected by immunofluorescence staining. Magnification, $\mathrm{x} 200 .{ }^{*} \mathrm{P}<0.05$ and ${ }^{* * *} \mathrm{P}<0.001$ vs. Oe-NC; ${ }^{\#} \mathrm{P}<0.01$ vs. Oe-OTUD6B-AS1+mimic-NC. OTUD6B-AS1, ovarian tumor domain containing 6B antisense RNA1; NC, negative control; Oe, overexpression; miR-3171, microRNA-3171; OD, optical density.

markedly upregulated in CRC cell lines compared with in the HIEC cell line, particularly in HCT116 cells (Fig. 4B). The miR-3171 level was markedly elevated following transfection with miR-3171 mimic (Fig. 4C). The dual-luciferase reporter assay demonstrated the binding of miR-3171 and OTUD6B-AS1, since the miR-3171 mimic + OTUD6B-AS1 WT group exhibited lower luciferase activity compared with the mimic-NC + OTUD6B-AS1 WT group (Fig. 4D). As expected, the results of the RT-qPCR assay (Fig. 4E) indicated that miR-3171 expression was markedly reduced following OTUD6B-AS1 overexpression compared with that in the OE-NC group. These results suggested that miR-3171 is a direct target of OTUD6B-AS1.
OTUD6B-AS1 suppresses the proliferation, invasion and migration of HCT116 cells by targeting miR-3171. To determine whether OTUD6B-AS1 can exert its effects on proliferation, invasion and migration of HCT116 cells by targeting miR-3171, a series of functional experiments was performed. As shown in Fig. 5A-C, co-transfection of OTUD6B-AS1 overexpression plasmid and miR-3171 mimics reversed the inhibitory effects of OTUD6B-AS1 overexpression alone on the proliferation, colony formation abilities and the Ki67 expression of HCT116 cells. Furthermore, the addition of miR-3171 mimics attenuated the effects of OTUD6B-AS1 overexpression on the invasive and migratory abilities of HCT116 cells (Fig. 6A-D). 
A
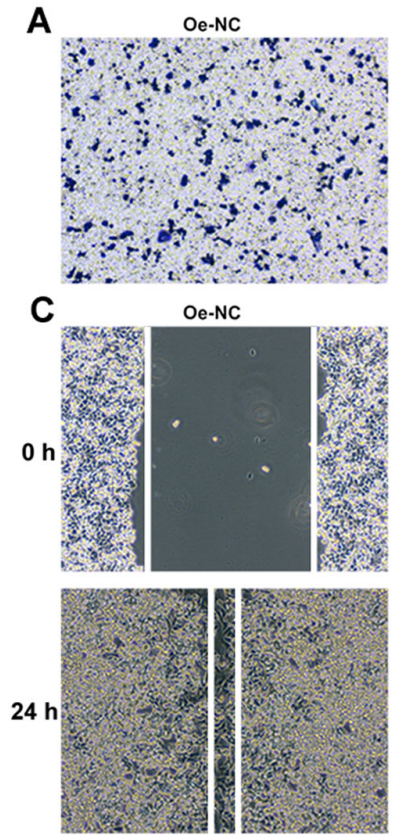

E

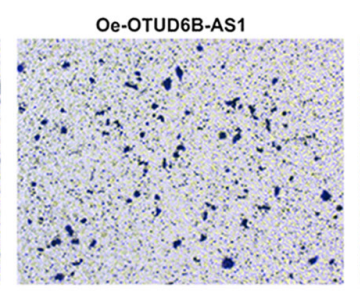

Oe-OTUD6B-AS1
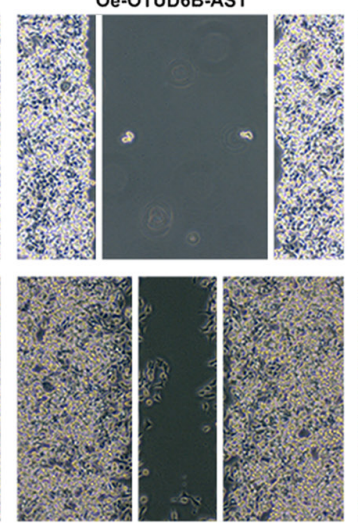

Oe-OTUD6B-AS1+mimic-NC

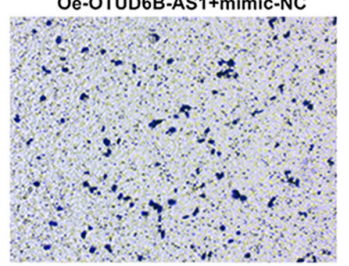

Oe-OTUD6B-AS1+mimic-NC
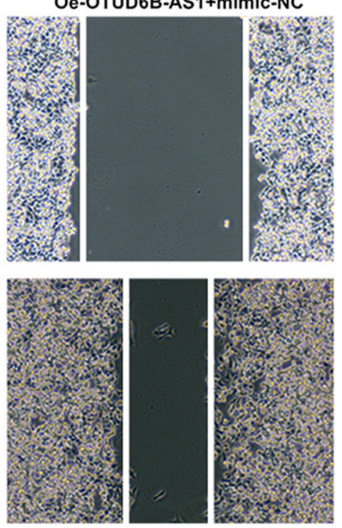

Oe-OTUD6B-AS1+miR-3171 mimic

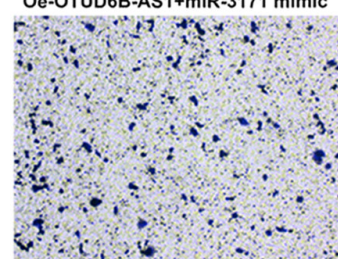

Oe-OTUD6B-AS1+miR-3171 mimic
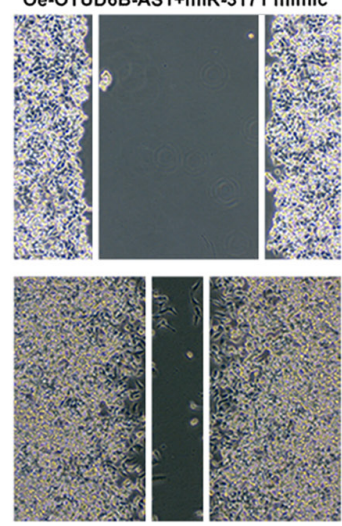

MMP-9

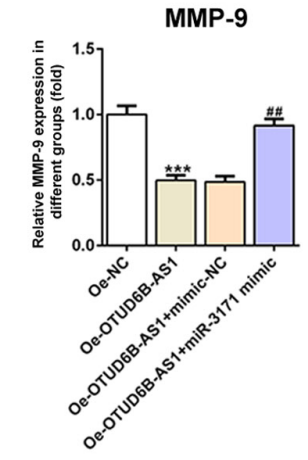

B

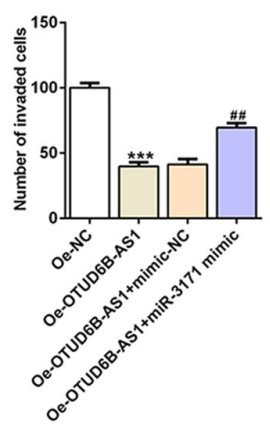

D

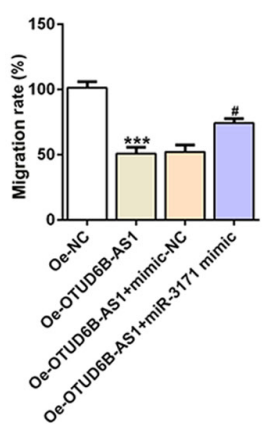

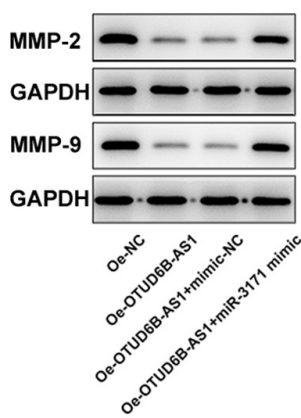

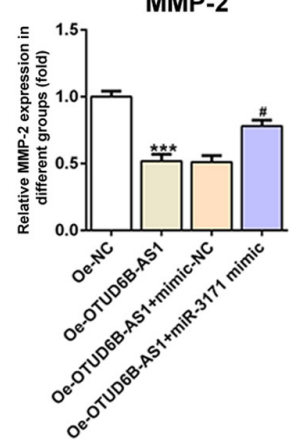

Figure 6. miR-3171 overexpression alleviates the inhibitory effects of OTUD6B-AS1 overexpression on the invasion and migration of HCT116 cells. (A) Invasive ability of HCT116 cells detected using a Transwell assay. Magnification, x100. (B) Relative number of invaded cells. (C) Representative images and (D) relative quantification of cell migration, as examined using a scratch assay. Magnification, x100. (E) Expression levels of migration-related proteins were examined by western blotting. All band images presented were obtained from the same representative gel. ${ }^{* * *} \mathrm{P}<0.001$ vs. Oe-NC; ${ }^{\#} \mathrm{P}<0.05$ and ${ }^{\# \#} \mathrm{P}<0.01$ vs. Oe-OTUD6B-A S1+mimic-NC. OTUD6B-AS1, ovarian tumor domain containing 6B antisense RNA1; NC, negative control; Oe, overexpression; miR-3171, microRNA-3171.

Additionally, the expression levels of migration-associated proteins were elevated when the HCT116 cells with overexpression of OTUD6B-AS1 were co-transfected with miR-3171 mimic (Fig. 6E). These results indicated that OTUD6B-AS1 inhibited the proliferation, invasion and migration of HCT116 cells by targeting miR-3171.

\section{Discussion}

CRC is the third most common type of cancer worldwide, and is a leading cause of mortality that threatens tens of thousands of lives $(22,23)$. During the initiation and development of CRC, various germline and somatic mutations assemble (24). With the help of advanced technology for the screening and treatment of CRC, the number of individuals $>50$ years old who are suffering from CRC is lower than several decades ago; however, the incidence is rising in young patients $(25,26)$. Due to the low survival rate and complex molecular mechanism underlying CRC tumorigenesis, it is necessary to identify novel and effective biomarkers for the treatment of the disease.
lncRNAs serve as important regulators in diverse physiological and pathological processes (27). Previous studies have revealed that lncRNAs are involved in all stages of carcinogenesis and tumor progression, such as tumor growth, tumor metastasis and tumor angiogenesis $(6,28)$. Several lncRNAs have been demonstrated to be associated with the entire process of CRC development. For example, glycolysis-associated lncRNA of colorectal cancer serves as an oncogene in colorectal carcinogenesis, and it can stabilize c-Myc to exert its promoting effect on CRC and glucose metabolism (26). Colorectal cancer-associated lncRNA could stimulate CRC progression via activation of the Wnt/ $\beta$-catenin signaling pathway by suppressing the activator protein $2 \alpha$ (29). HOX transcript antisense RNA, which is highly expressed in a large number of cancer cells, can regulate the methylation levels of histone H3K27, thus contributing to CRC development (30). These IncRNAs with abnormal expression in different phases of tumors could be potential diagnostic and prognostic markers (31). A previous study has demonstrated that overexpression of OTUD6B-AS1 could inhibit the proliferation, invasion and migration of renal clear cell 
carcinoma cells (11). When analyzing data from TCGA, it was revealed that the levels of OTUD6B-AS1 in CRC tissues were notably decreased compared with those in the adjacent non-tumor tissues, suggesting a potential antitumor effect of OTUD6B-AS1 in CRC progression. It is well-known that abnormal and uncontrolled proliferation is a characteristic of cancer cells (32). Furthermore, invasion and migration are considered to be two dominant processes for tumor metastasis, which leads to cancer-associated mortality (33). Thus, interruption of the aforementioned processes is an effective method for the inhibition of cancer metastasis. Therefore, the present study aimed to investigate whether OTUD6B-AS1 can have an effect on the proliferation, invasion and migration of CRC cells. The present study demonstrated that OTUD6B-AS1 expression was decreased in CRC cells compared with in a normal intestinal epithelial cell line, which was in accordance with the results of TCGA database analysis. Furthermore, overexpression of OTUD6B-AS1 inhibited the proliferation, migration and invasion of CRC cells.

Evolutionarily conserved miRNAs can suppress gene expression at the posttranscriptional level (34). Furthermore, they are considered to mediate the expression levels of $\geq 30 \%$ of all protein-coding genes, and are involved in most cellular processes (35). Over the past few decades, the aberrant expression profile of miRNAs has been frequently recognized in the peripheral blood and tumor tissue specimens from patients with CRC, hinting at the oncogenic effects of miRNAs in various types of cancer $(36,37)$. The upregulation of miR-135b has been commonly detected in patients with CRC by functional screening, and is associated with the clinical stage and cancer-specific survival of patients (38). Additionally, increased expression levels of miR-31 in patients with CRC are associated with poor prognosis (39). It has been hypothesized that the inhibition of dysregulated miRNAs could decrease the activity of CRC cells (40). In the present study, a binding site between OTUD6B-AS1 and miR-3171 was predicted using the Starbase database, and miR-3171 expression was upregulated in HCT116 cells. Therefore, it was hypothesized that OTUD6B-AS1 could inhibit CRC progression via inhibition of miR-3171 expression. To further verify this hypothesis, another RT-qPCR assay was performed and revealed that miR-3171 expression was markedly reduced following OTUD6B-AS1 overexpression. In a series of assays, it was observed that the additional treatment with miR-3171 mimics following OTUD6B-AS1 overexpression in HCT116 cells promoted the proliferation, invasion and migration of HCT116 cells, suggesting that OTUD6B-AS1 overexpression inhibited the proliferation, invasion and migration of HCT116 cells via downregulation of miR-3171 expression.

In conclusion, to the best of our knowledge, the present study was the first to investigate the role of OTUD6B-AS1 in CRC cells, and to reveal that IncRNA OTUD6B-AS1 overexpression inhibited the proliferation, invasion and migration of HCT116 cells, at least partially, by targeting miR-3171. Therefore, OTUD6B-AS1 may serve as a potential novel biomarker and target for the diagnosis and treatment of CRC. However, the use of only one CRC cell line in the cell function experiments and mechanism experiments, and lack of data obtained from clinical samples and investigations in animal models were limitations of the present study. Therefore, a comprehensive analysis is required in the future. Additionally, the use of only one TCGA dataset to investigate OTUD6B-AS1 expression is another limitation of the present study, and future studies should improve upon this and the other limitations.

\section{Acknowledgements}

Not applicable.

\section{Funding}

No funding was received.

\section{Availability of data and materials}

The datasets used and/or analyzed during the current study are available from the corresponding author on reasonable request.

\section{Authors' contributions}

WW and XC searched the literature, designed the experiments and performed the experiments. WW and JZ analyzed the data and wrote the manuscript. JZ revised the manuscript. All authors read and approval the final manuscript.

\section{Ethics approval and consent to participate}

Not applicable.

\section{Patient consent for publication}

Not applicable.

\section{Competing interests}

The authors declare that they have no competing interests.

\section{References}

1. Kuipers EJ, Grady WM, Lieberman D, Seufferlein T, Sung JJ, Boelens PG, van de Velde CJ and Watanabe T: Colorectal cancer. Nat Rev Dis Primers 1: 15065-15065, 2015.

2. Arnold M, Sierra MS, Laversanne M, Soerjomataram I, Jemal A and Bray F: Global patterns and trends in colorectal cancer incidence and mortality. Gut 66: 683-691, 2017.

3. Zhou LL, Zou MD and Li WM: Recent advances in colorectal cancer-specific nucleic acid aptamers for diagnostic and therapeutic applications. Sci Adv Mater 12: 38-43, 2020.

4. The Lancet Oncology: Colorectal cancer: A disease of the young? Lancet Oncol 18: 413, 2017.

5. Kasi PM, Shahjehan F, Cochuyt JJ, Li Z, Colibaseanu DT and Merchea A: Rising proportion of young individuals with rectal and colon cancer. Clin Colorectal Cancer 18: e87-e95, 2019.

6. Xu M, Xu X, Pan B, Chen X, Lin K, Zeng K, Liu X, Xu T, Sun L, Qin J, et al: LncRNA SATB2-AS1 inhibits tumor metastasis and affects the tumor immune cell microenvironment in colorectal cancer by regulating SATB2. Mol Cancer 18: 135, 2019.

7. Quinn JJ and Chang HY: Unique features of long non-coding RNA biogenesis and function. Nat Rev Genet 17: 47-62, 2016.

8. Rathinasamy B and Velmurugan BK: Role of lncRNAs in the cancer development and progression and their regulation by various phytochemicals. Biomed Pharmacother 102: 242-248, 2018.

9. Takata M, Pachera E, Frank-Bertoncelj M, Kozlova A, Jüngel A, Whitfield ML, Assassi S, Calcagni M, de Vries-Bouwstra J, Huizinga TW, et al: OTUD6B-AS1 might be a novel regulator of apoptosis in systemic sclerosis. Front Immunol 10: 1100, 2019. 
10. Wang Z, Xia F, Feng T, Jiang B, Wang W and Li X: OTUD6B-AS1 inhibits viability, migration, and invasion of thyroid carcinoma by targeting miR-183-5p and miR-21. Front Endocrinol (Lausanne) 11: $136,2020$.

11. Wang G, Zhang ZJ, Jian WG, Liu PH, Xue W, Wang TD, Meng YY, Yuan C, Li HM, Yu YP, et al: Novel long noncoding RNA OTUD6B-AS1 indicates poor prognosis and inhibits clear cell renal cell carcinoma proliferation via the Wnt/ $\beta$-catenin signaling pathway. Mol Cancer 18: 15, 2019.

12. Mahesh G and Biswas R: MicroRNA-155: A master regulator of inflammation. J Interferon Cytokine Res 39: 321-330, 2019.

13. Ambros V: The functions of animal microRNAs. Nature 431: 350-355, 2004

14. Wu Y, Yang L, Yu M and Wang J: Identification and expression analysis of microRNAs during ovule development in rice (Oryza sativa) by deep sequencing. Plant Cell Rep 36: 1815-1827, 2017.

15. Rane S, Sayed D and Abdellatif M: MicroRNA with a MacroFunction. Cell Cycle 6: 1850-1855, 2007.

16. Iorio MV and Croce CM: MicroRNA dysregulation in cancer: Diagnostics, monitoring and therapeutics. A comprehensive review. EMBO Mol Med 9: 852, 2017.

17. Wei Y, He R, Wu Y, Gan B, Wu P, Qiu X, Lan A, Chen G, Wang Q, Lin X, et al: Comprehensive investigation of aberrant microRNA profiling in bladder cancer tissues. Tumour Biol 37: 12555-12569, 2016.

18. Wang Z, Zhao Y, Wang Y and Jin C: Circular RNA circHIAT1 inhibits cell growth in hepatocellular carcinoma by regulating miR-3171/PTEN axis. Biomed Pharmacother 116: 108932, 2019.

19. Guo S, Zhu KX, Yu WH, Wang T, Li S, Wang YX, Zhang CC, GuoJQ, et al: SH3PXD2A-AS1/miR-330-5p/UBA2 ceRNAnetwork mediates the progression of colorectal cancer through regulating the activity of the Wnt/beta-catenin signaling pathway. Environ Toxicol: Oct 19, 2020 (Epub ahead of print). doi: 10.1002/tox.23038.

20. Livak KJ and Schmittgen TD: Analysis of relative gene expression data using real-time quantitative PCR and the 2(-Delta Delta C(T)) method. Methods 25: 402-408, 2001.

21. Li JH, Liu S, Zhou H, Qu LH and Yang JH: starBase v2.0 Decoding miRNA-ceRNA, miRNA-ncRNA and protein-RNA interaction networks from large-scale CLIP-Seq data. Nucleic Acids Res 42: D92-D97, 2014.

22. Torre LA, Bray F, Siegel RL, Ferlay J, Lortet-Tieulent J and Jemal A: Global cancer statistics, 2012. CA Cancer J Clin 65: 87-108, 2015.

23. Hua RX, Zhuo ZJ, Zhu J, Zhang SD, Xue WQ, Zhang JB, Xu HM, Li XZ, Zhang PF, He J, et al: XPG Gene Polymorphisms contribute to colorectal cancer susceptibility: A two-stage case-control study. J Cancer 7: 1731-1739, 2016.

24. He D, Ma L, Feng R, Zhang L, Jiang Y, Zhang Y and Liu G: Analyzing large-scale samples highlights significant association between rs10411210 polymorphism and colorectal cancer. Biomed Pharmacother 74: 164-168, 2015.

25. Global Burden of Disease Cancer Collaboration; Fitzmaurice $C$ Allen C, Barregard L, Bhutta ZA, Brenner H, Dicker DJ, ChimedOrchir O, Dandona R, Dandona L, Fleming T, et al: Global, regional, and national cancer incidence, mortality, years of life lost, years lived with disability, and disability-adjusted life-years for 32 cancer groups, 1990 to 2015: A Systematic Analysis for the Global Burden of Disease Study. JAMA Oncol 3: 524-548, 2017.

26. Tang J, Yan T, Bao Y, Shen C, Yu C, Zhu X, Tian X, Guo F, Liang Q, Liu Q, et al: LncRNA GLCC1 promotes colorectal carcinogenesis and glucose metabolism by stabilizing c-Myc. Nat Commun 10: 3499, 2019.
27. Heo JB, Lee YS and Sung S: Epigenetic regulation by long noncoding RNAs in plants. Chromosome Res 21: 685-693, 2013.

28. Zhang H, Chen Z, Wang X, Huang Z, He Z and Chen Y: Long non-coding RNA: A new player in cancer. J Hematol Oncol 6: 37, 2013.

29. Ma Y, Yang Y, Wang F, Moyer MP, Wei Q, Zhang P, Yang Z, Liu W, Zhang H, Chen N, et al: Long non-coding RNA CCAL regulates colorectal cancer progression by activating Wnt $/ \beta$-catenin signalling pathway via suppression of activator protein $2 \alpha$. Gut 65: 1494-1504, 2016

30. Cai B, Song XQ, Cai JP and Zhang S: HOTAIR: A cancer-related long non-coding RNA. Neoplasma 61: 379-391, 2014.

31. Galamb O, Barták BK, Kalmár A, Nagy ZB, Szigeti KA, Tulassay Z, Igaz P and Molnár B: Diagnostic and prognostic potential of tissue and circulating long non-coding RNAs in colorectal tumors. World J Gastroenterol 25: 5026-5048, 2019.

32. Wang AH, Fan WJ, Fu L and Wang XT: LncRNA PCAT-1 regulated cell proliferation, invasion, migration and apoptosis in colorectal cancer through targeting miR-149-5p. Eur Rev Med Pharmacol Sci 23: 8310-8320, 2019.

33. Zhang H, Song Y, Yang C and Wu X: Overexpression of lncRNA TUSC7 reduces cell migration and invasion in colorectal cancer. Oncol Rep 41: 3386-3392, 2019.

34. Lin J, Chuang CC and Zuo L: Potential roles of microRNAs and ROS in colorectal cancer: Diagnostic biomarkers and therapeutic targets. Oncotarget 8: 17328-17346, 2017.

35. Liu Z, Wang Y, Borlak J and Tong W: Mechanistically linked serum miRNAs distinguish between drug induced and fatty liver disease of different grades. Sci Rep 6: 23709, 2016.

36. Gmerek L, Martyniak K, Horbacka K, Krokowicz P, Scierski W, Golusinski P, Golusinski W, Schneider A and Masternak MM: MicroRNA regulation in colorectal cancer tissue and serum. PLoS One 14: e0222013, 2019.

37. Nagy ZB, Barták BK, Kalmár A, Galamb O, Wichmann B, Dank M, Igaz P, Tulassay Z and Molnár B: Comparison of circulating miRNAs expression alterations in matched tissue and plasma samples during colorectal cancer progression. Pathol Oncol Res 25: 97-105, 2019.

38. Valeri N, Braconi C, Gasparini P, Murgia C, Lampis A, Paulus-Hock V, Hart JR, Ueno L, Grivennikov SI, Lovat F, et al: MicroRNA-135b promotes cancer progression by acting as a downstream effector of oncogenic pathways in colon cancer. Cancer Cell 25: 469-483, 2014.

39. Sun D, Yu F, Ma Y, Zhao R, Chen X, Zhu J, Zhang CY, Chen J and Zhang J: MicroRNA-31 activates the RAS pathway and functions as an oncogenic MicroRNA in human colorectal cancer by repressing RAS p21 GTPase activating protein 1 (RASA1). J Biol Chem 288: 9508-9518, 2013.

40. Yin Y, Yan ZP, Lu NN, Xu Q, He J, Qian X, Yu J, Guan X, Jiang BH and Liu LZ: Downregulation of miR-145 associated with cancer progression and VEGF transcriptional activation by targeting N-RAS and IRS1. Biochim Biophys Acta 1829: 239-247, 2013.

This work is licensed under a Creative Commons Attribution-NonCommercial-NoDerivatives 4.0 International (CC BY-NC-ND 4.0) License. 